\title{
Interview Marc Faber
}

Marc Faber ist meistens anderer Meinung als die Mehrheit der Börsenexperten. Da der Schweizer erst über Risiken spricht, bevor er die Chancen an der Börse würdigt, wird er auch "Dr. Doom" genannt. Sein Börsenbrief, mit dem er die Anleger immer wieder konfrontiert, heißt "Gloom, Boom \& Doom Report". Mit 24 Jahren promovierte Faber im Fach Wirtschaftswissenschaft. Früh zog es ihn nach Asien. Faber erläutert die Grenzen von Börsenprognosen und bezeichnet die wachsende Börsenspekulation als gefährlich. „Sie ist ein Indiz vergangener Prosperität."

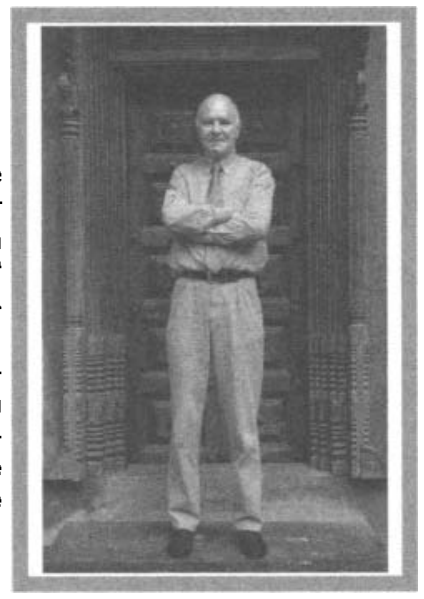

FRAGE: Herr Faber, Sie gelten als Investment-Guru, der in die Zukunft schauen kann. Erfüllt Sie soviel Zutrauen mit Stolz?

FABER: Ich halte es für unwichtig. Kein Mensch behauptet von sich selbst, er sei ein Guru. Es ist das Publikum, das einen dafür auswählt.

Aber es ist doch nicht unangenehm ein solches Publikum zu haben. Schließlich wird man an der Eitelkeit gepackt, und da stellen Sie das Gurutum in Frage?

An den Finanzmärkten gibt es jedes Jahr einen Aktienfonds mit einem Fondsmanager, der besonders gut abgeschnitten hat. Das ist erfreulich. Nur ist es selten so, dass im Jahr darauf derselbe Fondsmanager wieder an der Spitze steht. Es gibt überhaupt keine Beständigkeit verlässlicher Prognosen - aller Qualitäten der Geldverwalter zum Trotz. Der Amerikaner Joe Granville galt in den siebziger Jahren als Guru. Es war unglaublich, wie treffsicher er war. Der Mann setzte Kauf- und Verkaufs- 
signale für den Markt. Die Börse schien blind umzusetzen, was er prophezeite.

Das konnte natürlich nicht so weitergehen, irgendwann musste Granvilles ,Fähigkeit" an Schub verlieren?

Genau, und bei ihm ging es dann auch sehr schnell. Plötzlich war Schluss. Von einem Tag auf den anderen. Er verpasste die Hausse nach 1982. Einfach so. Die Seherqualitäten waren erschöpft. Seit den achtziger Jahren spricht niemand mehr von Granville. Ein Guru zu sein, ist ein vergängliches Geschäft.

Aber es scheint so, als ob die Börse diese Gurus trotzdem braucht, erst um sie zu hofieren und dann, um sie abzusägen. Schließlich gibt es in der Geschichte viele solcher Beispiele.

Der US-Amerikaner Robert Prechter ist auch so ein Fall. Ein brillanter Mann, dessen Bücher ich auch gelesen habe. Prechter prophezeite 1977, dass der Dow Jones Index auf 2300 Punkte steigen würde, und 1980, dass der Dow auf 2700 Punkte zulegt - und damals lag der Dow Jones bei rund 1000 Punkten. Im Jahr 1987 waren seine Prophezeiungen erfüllt. Prechter hatte einen guten Ruf, hatte Ansehen und nichts und niemand schien ihm etwas anhaben zu können...

\section{...bis auf die Börse selbst}

$\mathrm{Ja}$, bis er vom Börsencrash im selben Jahr wieder auf den Boden der Tatsachen zurückgeholt wurde. Nach dem Crash folgte die Hausse von 1988 bis zum Jahre 2000. Auch die verpasste er, sprich er sah sie nicht voraus.

Gurus sind also keine guten Ratgeber. Man sollte nicht auf sie hören?

Niemand weiß, was in der Zukunft passieren wird. Aber als Ökonom kann man grundsätzlich sagen, dass die Weltwirtschaft real mit drei Prozent pro Jahr wächst. Manchmal können es zwei, manchmal auch vier Prozent sein, wenn es aufgrund der Inflation nominal höher ausfällt. Es ist jedoch unmöglich, dass die Gesellschaft bis in alle Ewigkeit schneller wächst als das Bruttoinlandsprodukt. 
Was heißt das genau?

Wenn der Software-Konzern Microsoft jedes Jahr um 20 Prozent wächst, während die US-Wirtschaft nur 4 Prozent zulegt, dann wäre Microsoft irgendwann die gesamte US-Wirtschaft. Und das geht natürlich nicht. Microsoft wird also irgendwann so groß, dass der Konzern nicht mehr wesentlich schneller als die Gesamtwirtschaft wachsen kann. Solcherlei Aussagen über die Zukunft kann man treffen ohne ein Guru zu sein. Wagt man sich weiter vor, wird es gefährlich.

Von ihnen stammt folgendes Bonmot: Ein Anleger hätte in den vergangenen Jahrzehnten nur drei Anlageentscheidungen treffen müssen, um eine überdurchschnittliche Performance zu erreichen: Von 1970 bis 1980 hätte er in Gold, Öl oder Rohstoffen, von 1980 bis 1990 in japanischen Aktien und von 1990 bis 2000 in amerikanischen Aktien investiert sein müssen. Schön wäre es doch, wenn man solche langjährigen Trends frühzeitig erkennen würde?

$\mathrm{Ja}$, aber das ist sehr schwer. Allerdings lassen sich Indizien für einen neuen Trend manchmal erkennen, besonders wenn es von Zeit zu Zeit zu großen Übertreibungen kommt. Man sieht das, indem man die Preise der einen Vermögenswerte relativ zu anderen Vermögenswerten misst. Der amerikanische Dow Jones-Index lag 1980 bei rund 1000 Punkten, Gold kostete im selben Jahr 850 Dollar je Unze. Damals konnte man anhand der Preisbewegungen sagen, dass Gold teuer war und Aktien sowie Renten billig. An einer solchen Wegmarke kommt es zu Verschiebungen. Es kommt zu einer Veränderung der Leadership, wie wir es auch im Jahr 2000 erlebt haben. Damals konnte man erkennen, dass nicht mehr die Technologiebörse Nasdaq, sondern der Öl- und Minenbereich die Leadership übernehmen würden. Damals konnten wir ganz eindeutig sagen, dass Rohstoffe billig und Aktien teuer sind. Die Rohstoffpreise waren seit 1980 nominal und real sehr stark gefallen, während die Kurse von Aktien und Obligationen stiegen.

Mit einem solchen Ansatz lässt sich also ein neuer Investmenttrend identifizieren?

Bei derartiger Preisdiskrepanz ist es sehr wahrscheinlich, dass sich etwas ändert. Es ist ein Signal, dass die Blase platzt. Es gibt kein Rezept dafür, 
wie man den genauen Zeitpunkt der Korrektur bestimmt, man kann aber sicher sein: Diese Divergenz wird irgendwann korrigiert. Das muss so sein, aber man braucht mitunter Geduld.

\section{Wie lange kann diese Geduld strapaziert werden?}

Eine ganze Weile. Die Technologiebörse Nasdaq war meiner Meinung nach schon 1998 überbewertet, aber dennoch legte der Index noch bis zum Jahr 2000 zu. Erst danach ging es bergab. Es gibt viele solcher Übertreibungen. Im Jahr 1989 machte der japanische Nikkei-Index die Hälfte der weltweiten Börsenkapitalisierung aus. Japan war teurer als die USA, Großbritannien und Deutschland zusammen. Danach kam die Baisse. Übertreibungen werden irgendwann immer korrigiert, nach oben genauso wie nach unten.

\section{Was lernt man daraus?}

Wenn die ganze Welt dasselbe macht, sprich in dieselben Aktien oder Märkte investiert, dann ist das per Definition ein schlechtes Zeichen. Etwas hat sich in der Weltgeschichte schließlich nicht verändert, und das ist die Reichtumspyramide: Es gibt global gesehen immer noch wenige Reiche und viele Arme. Wenn alle Welt an eine Sache glaubt, und alle dort spekulieren, dann gibt es bald viele Arme.

\section{Wie erkennt man eine solche ungesunde Spekulationswelle?}

Erste Symptome sind hohe Handelsvolumina an der Börse. Aber auch wenn das Anlageobjekt jeden Tag in einer Börsensendung im Fernsehen besprochen wird, wenn so genannte Experten behaupten, nun werde eine neue Wirtschaftsära eingeleitet, wenn die Seminarangebote für Anleger Überhand nehmen, dann kann man davon ausgehen, dass wir eine Spekulationsblase haben.

Die Aktienblase ist im Jahr 2000 geplatzt. Es war ein langer Prozess. Hat sich danach eine andere Blase gebildet?

US-Immobilien werden jedes Jahr um zehn Prozent teurer, aber die Inflation und das Bruttoinlandsprodukt steigen nur um zwei Prozent. Die US-Bürger denken, wenn sie sich jetzt keine Immobilien kaufen, dann 
können sie es sich nie mehr leisten. Das treibt die Preise in ungesunde Höhen. Das Geld fließt mithin in unrentable Investitionen. Das hat in den USA zu einer starken Expansion der Verschuldung geführt, die seit 2000 um 9700 Milliarden Dollar angestiegen ist, während das Bruttoinlandsprodukt nur um 1800 Milliarden Dollar zugelegt hat. Die Schulden wachsen also schneller als das Wirtschaftswachstum. Der US-Konsum, Hauptantrieb der US-Wirtschaft, wird durch diese Immobilienblase angetrieben. Der Preisboom hat es erlaubt, die Immobilien zu refinanzieren und zu verbrauchen, während die realen Einkommen ja kaum gestiegen sind. Diese Situation ist sehr fragil.

Aber wie ist es möglich, dass an den Finanzmärkten solche Preisübertreibungen entstehen, schließlich gelten die Märkte doch als effizient?

Ein Finanzmarkt, der die Ersparnisse nimmt und diese durch Aktien oder Obligationen an die Unternehmen weiterleitet, ist ideal. Aber wenn alle Welt nur noch spekuliert und beispielsweise ein Konzern wie General Motors kein Geld mehr mit Autos verdient, sondern nur noch mit Finanzprodukten, dann ist das sehr bedenklich und nicht gerade Ausdruck einer gesunden Unternehmung.

\section{Können Sie das näher erläutern?}

Schaut man auf die Zusammensetzung der Gewinne der im USAktienindex Standard\&Poor's gelisteten Unternehmen, dann sieht man: Bis 1980 entstammten noch rund 40 Prozent der Gewinne dieser Konzerne aus der Fabrikation von greifbaren Gütern wie Autos, Kühlschränken oder Flugzeugen, aus dem Bereich eben, den man auch als Realwirtschaft bezeichnen kann. Heutzutage machen die Gewinne aus der Realwirtschaft im Standard\&Poor's nur noch etwa 10 Prozent aus, während die Gewinne aus dem Finanzsektor 50 Prozent beisteuern. Das sind inflationäre Gewinne durch Vermögensgüterinflation. Über die Realwirtschaft, wo Güter produziert und Dienstleistungen erbracht werden, hat sich eine gewaltige Finanzindustrie gestülpt, in der alle Akteure spekulieren. Das ist die eigentliche Blase. Wir, der Sektor in dem ich arbeite, sind die Blase. 
Und diese Blase entsteht durch einen Angebotsüberhang von Geld?

$\mathrm{Ja}$, auch. Die Funktion des Geldes ist die Wertkonservierung. 10.000 Euro auf der Bank sollten in zehn Jahren die gleiche Kaufkraft haben, plus Realzins von 1,5 bis 2 Prozent. Aber wenn die Zinsen so unglaublich künstlich tief gedrückt werden durch die Zentralbanken, wie es lange geschehen ist, dann gibt es eine Inflationsgefahr: Papiergeld ist in den letzten Jahren zunehmend entwertet worden, einfach dadurch dass die Geldmenge schneller gewachsen ist als die Wirtschaft, als das Bruttoinlandsprodukt. Zwar kosten Güter wie Faxmaschinen oder Handys immer weniger, doch Immobilien und Aktien sind enorm gestiegen in den letzten 20 Jahren, was immer mehr Akteure auf diesen Investitionspfad führte.

\section{Und das ist das Ergebnis angeblich effizienter Märkte?}

Ein Markt ist ein Markt. Es kann immer zu längeren Übertreibungsphasen kommen. Doch fest steht: Die heutige Weltwirtschaftslage kann langfristig nicht anhalten. Etwas wird passieren. Das Finanzsystem, insbesondere aufgrund der Derivativprodukte, der hohen Verschuldung und der relativ niedrigen Volatilität, ist sehr angreifbar geworden. Da muss kein großer Sturm kommen, um das Ungleichgewicht zu einem großen Problem zu machen.

Sie sprechen von Inflationsgefahr, doch die entsprechenden Indizes in den USA weisen kaum Preissteigerungen aus?

Der amerikanische CPI-Index basiert auf besonderen Messmethoden. Zum einen wird hedonisch justiert, das heißt, nominale Preiserhöhungen beispielsweise für ein TV-Gerät werden nach unten korrigiert, weil man davon ausgeht, dass ein Teil der höheren Preise Ausdruck höherer Qualität ist. Zum anderen macht etwa das Gesundheitswesen in den USA rund 16 Prozent des Bruttoinlandsprodukts aus, doch wird dieser Sektor bei der Inflationsmessung im CPI nur zu sechs Prozent berücksichtigt. Die Inflationsraten in den USA, die enormen Einfluss auf die Weltbörsen haben, sind also eigentlich höher als es ausgewiesen wird. 
Dennoch, Elektronikgeräte und vieles andere werden immer billiger.

Das stimmt, und deshalb sehen die meisten Anleger die Inflation auch nicht. Die Erhöhung der Geldmenge kann zu verschiedenen Symptomen führen: In den sechziger Jahren schlug sich die wachsende Geldmenge auf die Löhne nieder, in den siebziger Jahren stiegen die Rohstoffpreise. Seit 25 Jahren sehen wir durch die Geldmengenausweitung steigende Vermögenswerte für Aktien und Obligationen - auch das ist Inflation.

Und was sagen Sie den Experten, die vor einer Deflation in den USA warnen, was die Börsen nachhaltig negativ beeinflussen würde?

Natürlich kann es eines Tages zu einer Deflation kommen, dann nämlich, wenn der Schuldenberg in den USA zusammenbricht, weil sich das Wirtschaftswachstum verlangsamt. Zunächst wird die US-Zentralbank aber in einer solchen Situation Geld drucken, und wenn nötig mit dem Helikopter über dem Land abwerfen, was zunächst zu einer Hyperinflation führen könnte.

Wie mutiert eine solche Entwicklung in die Deflation?

Durch die Inflation des Schuldenbergs. Im Jahr 1980 machten die USSchulden 120 Prozent des Bruttoinlandsprodukts aus, jetzt liegt dieser Wert bei über 300 Prozent. Irgendwann wird der Berg größer, aber das Gelddrucken verliert an Wirkung und wird nicht mehr zum Wachstum beitragen, weil die Produktion und Investition in China und Asien stattfindet. Und dann platzt der Geldhaufen. Und es folgt die Deflation.

Halten wir fest, unsere globale Wirtschaft ist mit Geld überschwemmt, was zu einer Inflation bei Vermögensgütern geführt hat - vieles an den Börsen ist ihrer Meinung nach teuer?

Vieles ja, aber nicht unbedingt alles. Dadurch, dass viel Geld in die Übertreibungen bewegt wird, ist logischerweise anderes unterbewertet. Wenn man im Jahr 1999 eine Kaufempfehlung für Öl gegeben hätte, dann wäre der Kommentar damals gewesen: Öl fällt ja nur. Damals war die Aufmerksamkeit nur auf Neuen Markt konzentriert. Der war überbewertet bei gleichzeitigen Unterbewertungen der Unternehmen aus der Old Economy. 
Also gibt es auch heute Bereiche, wo es vergleichsweise billig ist, wo es sich mittelfristig lohnen kann zu investieren?

Agrarrohstoffe wie Weizen, Mais und Soja sind relativ billig. Das Risiko, dass die Preise sinken, schätze ich auf 20 Prozent. Die zunehmende Anzahl von Dürren in Asien könnte zu großen Preisausschlägen nach oben führen. Es ist gut möglich, dass die Nahrungsmittelpreise binnen drei Jahre sehr stark ansteigen. Zudem ist Gold im Vergleich zu amerikanischen Aktien billig. Falls aufgrund des massiven Gelddruckens durch die US-Zentralbank der Dow Jones auf 36.000 Punkte steigen sollte, so dürfte auch der Preis für die Unze Gold auf Minimum 3.600 Dollar steigen.

Das ist ihre Prognose, wenn Sie Recht haben, dann werden die GuruRufe wieder lauter.

Wie gesagt, niemand kann die Zukunft vorhersehen. Nehmen Sie Asien. Klar gibt es hier Chancen, aber es gibt hier auch geopolitische Risiken. Es kann zu Kriegen kommen, was die Märkte durchschütteln würde. Oder ein Risiko wie die Vogelgrippe Sars. Als das Virus erstmals die Runde machte, da ist der Tourismus in Asien völlig zusammengebrochen. In den Luxushotels von Hongkong war urplötzlich kaum noch ein Bett besetzt. Man sieht hier sehr deutlich, von wie vielen Variablen steigende Aktienkurse abhängen.

Man hat das Gefühl, dass sich immer mehr Menschen auf das Spiel mit den Aktien einlassen wollen. Die Umsätze steigen und steigen. Kann das so weitergehen? Welche Rolle spielt die Börse in der Zukunft?

In den siebziger Jahren betrug das tägliche Aktienvolumen an dem New York Stock Exchange rund 12 Millionen, und dies an einem guten Tag. Heute werden täglich etwa 1.2 Milliarden Aktien an der New Yorker Börse gehandelt und oft ein noch größeres Volumen an der Technologiebörse Nasdaq. Dieses exponentiale Wachstum kann langfristig nicht so weitergehen. Der gesamte Finanzsektor wird eines Tages gewaltig schrumpfen. Die derzeitige Finanzblase ist ja nicht nur an den Aktienbörsen messbar, sondern auch bei Bonds, Derivaten und strukturierten Produkten. Das Devisenmarktvolumen ist mit täglich weit über 1000 Milliarden Dollar wesentlich größer als das eigentliche Handelsvolumen 
der Güter. Die Verdienstmöglichkeiten eines Hedgefonds-Managers sind enorm - je mehr der Geldspiegel steigt, desto mehr Kunden hat man als Geldverwalter. Ich habe davon auch profitiert. Doch all das ist ungesund.

\section{Sind Sie ein Spekulant?}

Ich bin grundsätzlich gegen die Spekulation. Wenn es überall in der Welt solche Spekulationswellen gibt, dann ist das kein Zeichen künftiger Prosperität, sondern ein Indiz für vergangene Prosperität. Es ist eine Phase, in der Geld gedruckt wird, um diesen geschaffenen Reichtum und Wohlstand zu erhalten.

Was ist zu tun? Soll die Politik einschreiten durch neue Regelungen?

Ich bin für freie Märkte, eine Regierung sollte nicht einschreiten, auch nicht, wenn es schlecht läuft. Insbesondere sollte eine Regierung nicht mit Gelddrucken und einer expansiven Geldpolitik mit künstlich tiefen Zinssätzen, wie es in den USA in den letzten Jahren der Fall war, die Wirtschaft zu stützen suchen. Das Schlimme ist jedoch, dass die Politik dann einschreitet, wenn große Fische betroffen sind. Es war nicht gut, dass der Hedgefonds LTCM im Jahr 1998 gerettet wurde. Nun nehmen die Märkte an, es gebe kein Risiko, und deshalb geht man immer höhere Risiken ein. Es ist das klassische „Moral Hazard“-Problem. Die Akteure gehen davon aus, dass die Politik ganz große Pleiten finanziell dann auffängt, wenn das Finanzsystem gefährdet ist. Und das erhöht natürlich den Spekulationsdrang. 